\title{
Simulation Design and Characteristics Analysis of Dedicated Dynamometer for Cold Roll-beating
}

\author{
Zhang Ruiyuan \\ School of Mechanical Instrumental Engineering \\ Xi'an University of Technology \\ Xi'an, China \\ E-mail: zry257640@163.com
}

\author{
Li yan \\ School of Mechanical Instrumental Engineering \\ Xi'an University of Technology \\ Xi'an, China \\ E-mail: jyxy-ly@xaut.edu.cn
}

\begin{abstract}
Cold roll-beating is a new advanced plastic forming technology, obtaining the forming force varying law is key to study the forming mechanism. Aiming at force measuring requirement in cold roll-beating forming process and the forming principle, a strain gage type dynamometer dedicated for cold roll-beating was designed, and a new type of elastomeric structure was proposed. ABAQUS software was used to simulate the static and dynamic characteristics of the structure, then the strain distribution rule of the elastomer surface sensitive position and the harmonic response characteristics and dynamic sensitivity of each gauging point were studied. Finally, experiment were carried out to calibrate the design dynamometer] and the results show that the dynamometer is suitable for measuring the cold roll-beating forming force.
\end{abstract}

Keyword-Cold roll-beating; Dynamometer; Surface strain ; Dynamic response; Calibration tests.

\section{INTRODUCTION}

Cold roll-beating is an advanced precision plastic forming process, mainly used in processing multiple spline, screw, etc. Rolling force and thermal-mechanical coupling are important parts of the cold roll-beating forming mechanism. At present, the researches on roll-beating force are mainly focused on the kinetics simulation and theoretical analysis ${ }^{[1][2]}$. It is necessary to design a kind of dynamometer dedicated for cold roll-beating to verify the simulation model, and further study the influence of various process parameters on the rolling force.

Considering the rolling force is of three-dimension, low frequency and high loading ${ }^{[3][4]}$, the elastomer with strain gauge pasted on is used in the dynamometer ${ }^{[5][6]}$. Elastomer is the key part of the strain type dynamometer, its static and dynamic characteristics will directly affect the overall performance of the dynamometer and the subsequent optimal design. Strain state on the sensitive surface of the elastomer is very complicated under the condition of three-dimensional force, which makes it difficult to accurately locate the gauge pasting position, and the force coupling problems cannot be ignored ${ }^{[7]-[9]}$.

In this paper, a elastomer with double octagonal ring is presented, and ABAQUS software is used to simulate and analyze the statics and dynamics of the structure, strain distribution rule of the elastomer surface sensitive position is studied, the reasonable pasting positions are determined, and also the dynamic response characteristics of each gauging point are obtained. Finally, through the experiment, the dynamometer is calibrated, and the dimensional coupling is reduced by decoupling matrix.

\section{FORCE MEASURING PRINCIPLE AND STRUCTURE OF THE ELASTOMER}

\section{A. Forece measuring principle of cold roll-beating}

In cold roll-beating forming process, rolling wheels (rollers) with certain shapes continuously roll and strike the work piece, which makes tiny plastic deformation occurring, with the relative motion relationship between the rolling wheel and the work piece; these tiny plastic deformations are accumulated to generate the required functional surface ${ }^{[4][10]}$.

The forming measuring principle of cold roll-beating is shown in the Fig .1, the rolling wheels rotate around the fixed point with high-speed, the work piece is installed on the dynamometer and feeds along the horizontal direction o realize the process of roll-beating. In the forming process, the strain gauges on the elastomer will change the strain signals into resistance signals, the later will be read by the recorder through the whole bridge circuit, then the force value will be obtained based on the calibration results and decoupling matrix.

\section{B. Structure of elasmoter}

The dynamometer structure mainly includes clamping part, elastomer, and pedestal, of which the elastomer is of integral structure, with double octagonal ring form, and its material is $40 \mathrm{Cr}$. Through the strength check calculation, under the measuring range of $7.5 \times 10^{3}$, the thick of octagonal ring is $6 \mathrm{~mm}$, for the convenience of patching, taking $25 \times 25 \mathrm{~mm}^{2}$ as edge dimensions. Fig .2 shows the simplified model of the octagonal ring and serial numbers of the main faces, of which the coordinate system is the unified coordinate system in this paper.

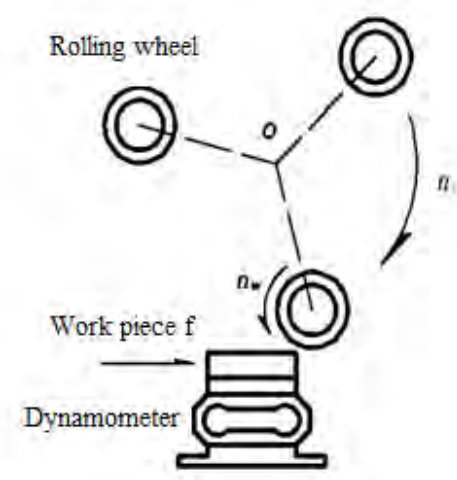

(a) Cold roll-beating principle 


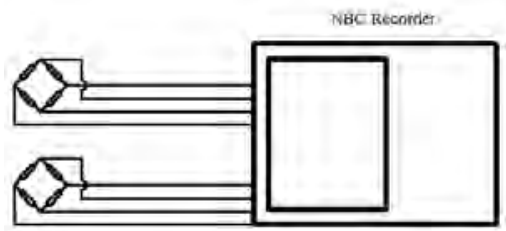

(b) NBC Recorder

Figure 1. Force measuring principle
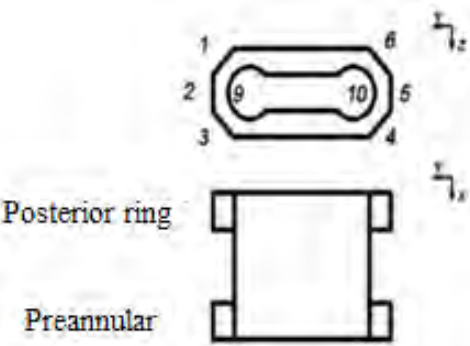

(a) Main view of octagonal ring
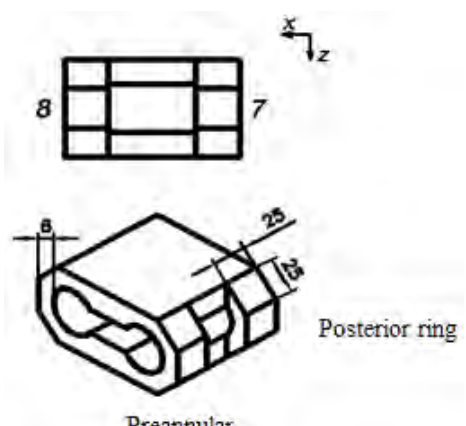

(b) Three-dimensional figure of the octagnal ring

Figure 2. Octagonal ring

\section{ESTABLISHMENT OF THE SIMULATION MODEL}

\section{A. Force loading}

The loading point of rolling force is selected in the marked circle location shown in the Fig .3, and the distance from the top center of the octagonal ring is $25 \mathrm{~mm}$ which is the distance from work piece surface to the top of octagonal ring. The position of working point will move along $\mathrm{Y}$ direction, while the top center is of good symmetry, which is advantageous for the analysis and comparison of each symmetric surfaces and verifying the accuracy of the simulation results.

Previous simulation results show that the rolling force is three-dimensional, the force along the groove depth direction is the largest, and the force perpendicular to groove depth symmetry surface is the smallest ${ }^{[3][4]}$, in this paper, $F_{z}>F_{y}>F_{x}$ and ranges of the three force are all $7.5 \times 10^{3} N$.

\section{B. $\quad$ Parameters setting}

The overall material of the dynamometer is $40 \mathrm{Cr}$, the bottom of dynamometer is constrained while simulation analysis, the type of elements are C3D4 and C3D8R, the element size of the strain surface is set as $3 \mathrm{~mm}$, and the others are all $20 \mathrm{~mm}$, the number of element is 21957 , and the meshing results is shown in the Fig .3.

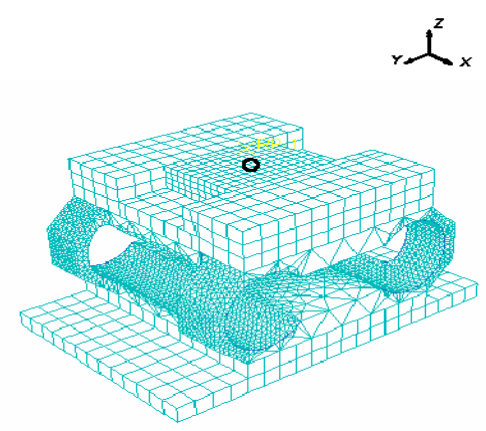

Figure 3. FEM model

\section{ANALYSIS OF THE SIMULATION RESULTS}

\section{A. Selection of each gauging point}

To select the gauging points reasonably, it is necessary to obtain the strain distribution rule of each sensitive surface. The strain components of surface strain along the normal are 0 , so the two-dimensional strain contours can be used to reveal the stain distribution rule of the octagonal ring sensitive surface. For the analysis methods are different at different direction, only taking example of direction $\mathrm{Z}$ with full scale loaded of $7.5 \times 10^{3} \mathrm{~N}$. Fig .4 shows surface strain distribution of the $2^{\#}, 5^{\#}$ ridge surfaces while direction $\mathrm{Z}$ is loaded full scales and the positions of $2^{\#}, 5^{\#}$ ridge surfaces on the octagonal ring.

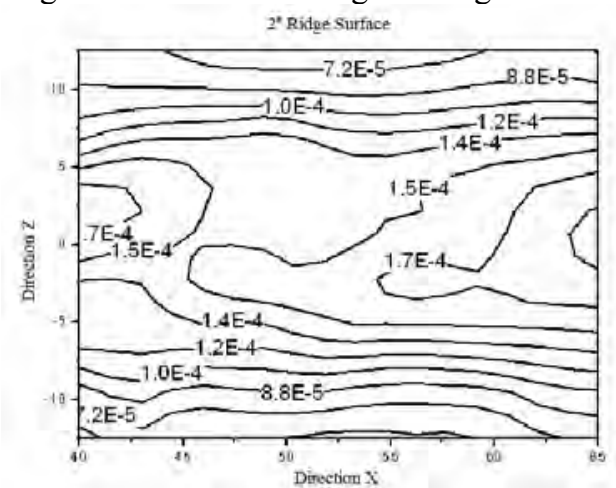

(a) Surface strain states of $2^{\#}$ ridge surface
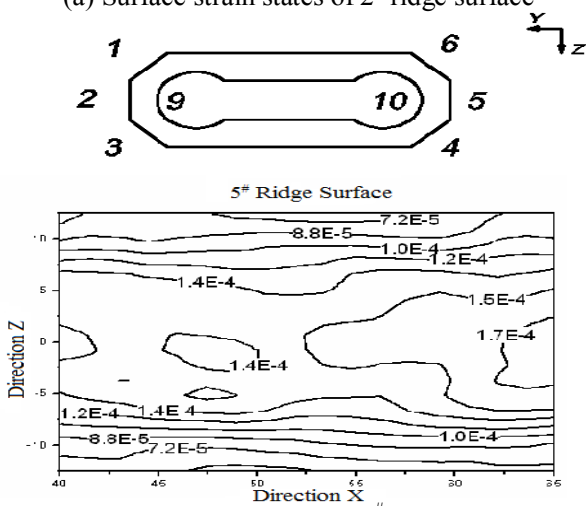

(b) Surface strain states of $5^{\#}$ ridge surface

Figure 4. Surface strain states of 2\#, 5\#ridge surface

As can be seen from the Fig .4, the surface strain distribution laws of $2^{\#}$ and $5^{\#}$ ridge surface which are in a symmetrical position under the condition of Z-direction loading are basically identical. $2^{\#}$ and $5^{\#}$ ridge surfaces sustained tensile stress and the biggest tension strain area 
is $170 \mu \varepsilon$, which appears at the position of the right side of ridge surface center and this position is suitable as gauging point of $\mathrm{Z}$-direction.

After the comprehensively analyzing the strain distribution of each surfaces under Z-direction loading, $2^{\#}$, $5^{\#}$ ridge surfaces with obvious tensile strain and $9^{\#}, 10^{\#}$ cylinders with obvious compressive strain are selected as the gauging point positions of Z-direction, and the rest of the surfaces with smaller strain can be pitched strain gauges of other directions to reduce the impact on the rest of the forces.

Table 1 shows the strain analysis results of sensitive surfaces along each direction, of which "+" represents tension strain and "." represents compressive strain, can be selected as the position of gauging point. The blank means inconspicuous strain and can't be chosen as gauging point.

TABLE I. THE STRAIN STATE OF SENSITIVE SURFACES

\begin{tabular}{|c|c|c|c|c|c|c|c|c|c|c|}
\hline & \multicolumn{10}{|c|}{ Surfaces } \\
\cline { 2 - 11 } & 1 & 2 & 3 & 4 & 5 & 6 & 7 & 8 & 9 & 10 \\
\hline $\mathrm{X}$ & & & & & & & - & + & & \\
\hline $\mathrm{Y}$ & + & & - & + & & - & & & & \\
\hline $\mathrm{Z}$ & & + & & & + & & & & - & - \\
\hline
\end{tabular}

\section{B. Harmonic response analysis}

Considering the loading frequency in actual working condition is under the range of $0 \sim 120 \mathrm{~Hz}$, which is much less than the lowest natural frequency of $523 \mathrm{~Hz}$, the harmonic excitation frequency can be chosen as $0,40,80$ and $120 \mathrm{~Hz}$ in the simulation process, the amplitude value is $7500 \mathrm{~N}$. Due to the rolling force along the X-direction is almost zero, only surface strain response under loading in the $\mathrm{Y}$ and $\mathrm{Z}$ directions are focused, where the surface strain response refers to the sum of each gauging point surface strain increment, Fig .5 shows the strain response amplitude changing with loading frequency .

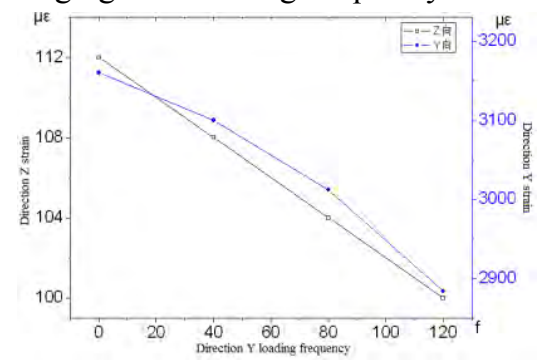

(a) Loading in direction $\mathrm{Y}$

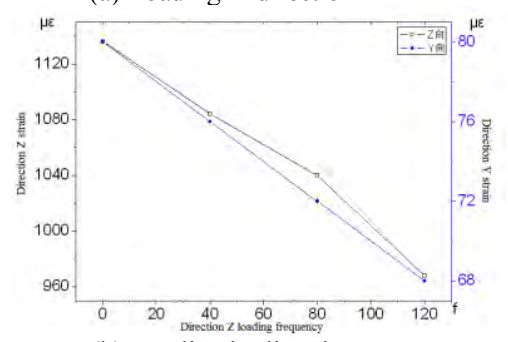

(b) Loading in direction $\mathrm{Z}$

Figure 5. Varing of strain response amplitude with loading frequency

From the Fig .5, it can be seen that, with frequency increasing from 0 to $120 \mathrm{~Hz}$, the harmonic response amplitude tends to decline, while loading in Y direction, the amplitude of $Y$ changes from $3160 \mu \varepsilon$ to $2884 \mu \varepsilon$ and amplitude of $\mathrm{Z}$ from $112 \mu \varepsilon$ to $100 \mu \varepsilon$, while loading in direction $\mathrm{Z}$, the amplitude of $\mathrm{Y}$ changes from $80 \mu \varepsilon$ to 68 $\mu \varepsilon$ and the amplitude of $Z$ from $1136 \mu \varepsilon$ to $968 \mu \varepsilon$.

\section{Dynamic sensitivity}

Dynamic sensitivity of Elastomer is defined as the ratio of a dynamic force amplitude and surface strain response; Fig .6 shows the dynamic sensitivity changing with loading frequency.

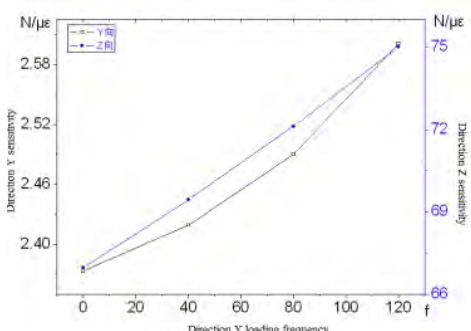

(a) Loading in direction $\mathrm{Y}$

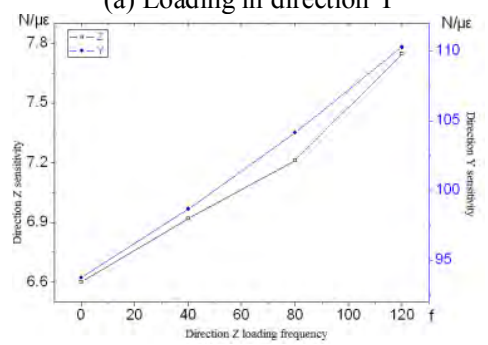

(b) Loading in direction $\mathrm{Z}$

Figure 6. Varing of dynamic sensitivity with loading frequency

From the Fig .6, it can be seen that, with loading frequency increasing, sensitivity is always increasing: while loading in $\mathrm{Y}$-direction, the sensitivity of $\mathrm{Y}$ changes from $2.37 \mathrm{~N} / \mu \varepsilon$ to $2.60 \mathrm{~N} / \mu \varepsilon$, and sensitivity of $Z$ from $66.96 \mathrm{~N} / \mu \varepsilon$ to $75 \mathrm{~N} / \mu \varepsilon$; while loading in direction $Z$, the sensitivity of $Y$ changes from $93.7 \mathrm{~N} / \mu \varepsilon$ to $110.3 \mathrm{~N} / \mu \varepsilon$ and sensitivity of $Z$ from $6.6 \mathrm{~N} / \mu \varepsilon$ to $7.7 \mathrm{~N} / \mu \varepsilon$.

\section{EXPERIMENTAL RESULTS}

\section{A. Static calibration and dynamic correction}

In the experiments direction $\mathrm{X}$ is not considered and only the directions $\mathrm{Y}$ and $\mathrm{Z}$ are calibrated and the form of whole bridge is selected as calibration circuit. Along each direction, the loading is increased step by step to $750 \mathrm{~kg}$, and $50 \mathrm{~kg}$ per step is selected. The output of signal recorder NEC - RA1200 is strain, the unit is $\mu \varepsilon$, each bridge voltage is $2 \mathrm{~V}$. Fig .7 shows the static calibration curves of direction of $\mathrm{Y}$ and $\mathrm{Z}$.

It can be calculated from the Fig .7 that while loading on the direction $\mathrm{Y}$, the sensitivity of direction $\mathrm{Y}$ is 0.25 $\mathrm{kg} / \mu \varepsilon$, while loading on the direction $Z$, the sensitivity of direction $Z$ is $0.69 \mathrm{~kg} / \mu \varepsilon$, compared with the simulation results, the error is within $7 \%$.

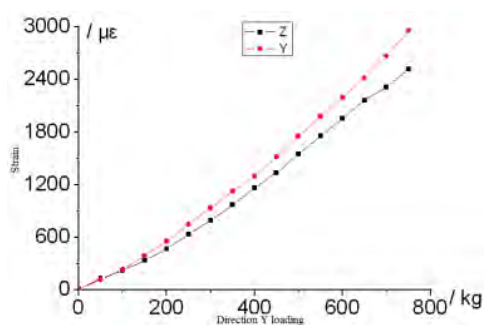

(a) Static calibration curves with loading in direction $\mathrm{Y}$ 


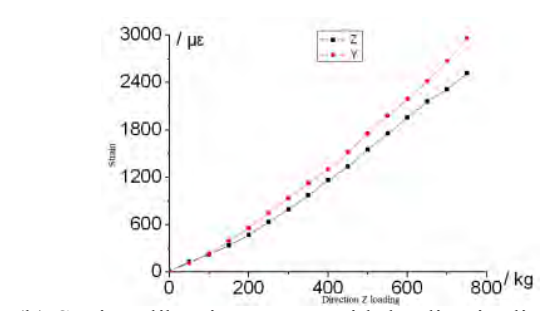

(b) Static calibration curves with loading in direction $\mathrm{Z}$

Figure 7. Static calibration curves

Considering the rolling force is of low frequency load, it is necessary to dynamically modify the sensitivity, the simulation results show that with the increase of loading frequency, the sensitivity is of linear increasing tendency, the corrected sensitivity can be expressed as follows:

$$
S=S^{\prime}+\Delta S \cdot f / 120
$$

Where, $f$ is the actual loading frequency; $S$ ' is the static sensitivity while $f=0 \mathrm{~Hz} ; \Delta S$ is the sensitivity increment within $0 \sim 120 \mathrm{~Hz}$.

\section{B. Actual roll-beating force measured}

The work-piece material used in cold roll-beating experiments is pure aluminum, and rolling wheel speed is $1200 \mathrm{rpm}$, the feeding speed is $490 \mathrm{~mm} / \mathrm{min}$, vertical rolling amount is $3 \mathrm{~mm}$, data sampling frequency is $500 \mu \varepsilon$. From the Fig .8 (a) and (b), it can be seen that when the roller rolling into the work piece, the rolling force is increasing gradually. From Fig .8(c), it also can be seen that the rolling force of direction $\mathrm{Z}$ is $3850 \mathrm{~N}$ in steady state, Fig .8(d) shows the experiment instrument.

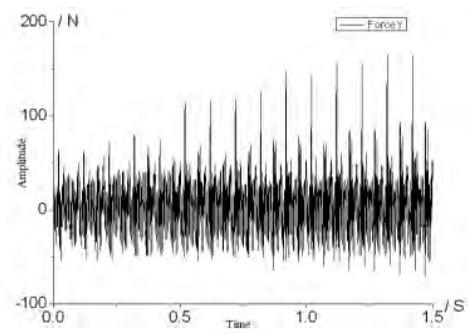

(a) Actual rolling force when beat into along direction $\mathrm{Y}$

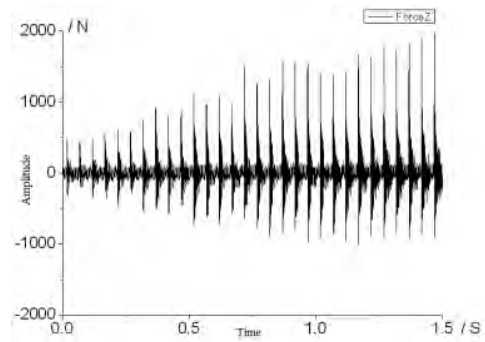

(b) Actual rolling force whiling beat into along direction $\mathrm{Z}$

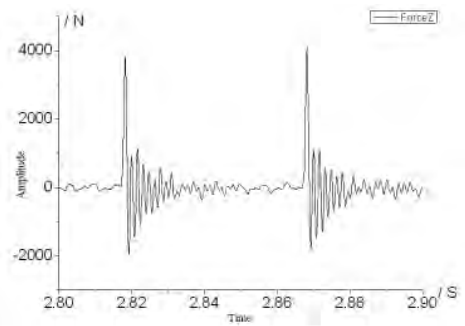

(c) Actual rolling force when $\mathrm{Z}$ direction in steady state

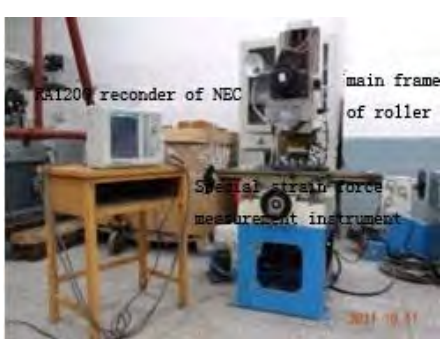

(d) Experiment instrument

Figure 8. Experimental result and instrument

\section{CONCLUSIONS}

In this paper, the finite element simulation technology was applied to the design process of strain type dynamometer dedicated for cold roll-beating forming, static and dynamic simulation analysis of was carried out by using ABAQUS, the surface strain distribution of elastomer sensitive spots, position of gauging point and dynamic response characteristic are obtained. Through experiments, the dynamometer was calibration. Cold rollbeating experiment results show that the dynamometer is suitable for measuring the forming force.

\section{ACKNOWLEDGMENT}

This project is supported by National Natural Science Foundation of China [Grant No. 51475366 and 51475146] and Key Laboratory of Scientific Research Projects of Shaanxi Educational Committee [Grant No. 12JS072].

\section{REFERENCES}

[1] J. H. Quan, F. K. Cui, J.X. Yang, H.Y. Xu, Y.J. X, “Numerical Simulation of Involute Spline Shaft's Cold-rolling Forming Based on ANSYS/LS-DYNA",China Mechanical Engineering, Wuhan, vol.19(4),pp. 419-422,Dec.2008.

[2] Y. Li,M. S. Yang,B. Li,S. J. Li,Q. L. Yuan, “Dynamics Simulation and Analysis of Lead Screw Cold Roll-Beating ",Journal of Xi'an University of Technology, Xi'an,vol.25(4),pp. 383-387,2009.

[3] Z.Y. Zhao, M.S. Yang, Q.L. Yuan, K. Zhao, "Deformation Force Simulation of Lead Screw Cold Roll beating Based on ABAQUS ", Foundry Technology, Xi'an,vol.32(8), pp. 1165- 1169,Dec.2011.

[4] M.S. Yang, Y. Li, Q.L. Yuan, “A Hybrid Method to Deformation Force of High-speed Cold Roll-beating Forming ",Journal of Digital Information Management,vol.11(2),pp. 146-153,April 2013.

[5] M.Meng,Y.J. Ge,Z.C. Wu,Y. Ge,M.Qian, "Design and Stimulation for a Novel Elastic Body of Multi-Axis Accelerometer",Sensors and Actuators,Nanjing,vol.16(4),Dec. 2003.

[6] B.Y. Wu,F. Shen,Z.C. Wu, "Study on Optimal Design Method for the Structure of Multi-Axis Force Strain Sensor",Sensors and Actuators, Nanjing,vol. 23(10), pp. $1412 \sim 1416$,Oct.2010.

[7] H. Takao, Y. Matsumoto, H. Seo, M. Ishida, T. Nakamura "Analysis and design considerations of three-dimensional vector accelerometer using SOI structure for wide temperature range", Sensors and Actuators,Nanjing,vol.55(8), pp. 91-97,July 1996.

[8] H. Seidel, H. Riedel, R. Kolbeck, G. Mück, W. Kupke, M Königer, "Capacitive Silicon Accelerometer with Highly Symmetrical Design”, Sensors and Actuators, Nanjing, vol.49(21), pp. 312-315, February 1990.

[9] J. W Joo, K. S Na, D. I Kang, “ Design and Evaluation of a Sixcomponent Load Cell”, Measurement, vol.32(2), pp.125-133, September 2002.

[10] M.S. Yang, Y. Li, Q.L. Yuan, Y.X. Li, R. Liang, "Theoretical Analysis and Experimental Study on Stripe of Slab Cold Rollbeating", Mechanical Science and Technology for Aerospace Engineering, Xi'an, vol. 32(9), pp.1363-1367, September 2013. 